\title{
ROLLING AND RECRYSTALLIZATION TEXTURES IN IRON-3\% SILICON
}

\author{
L. SEIDEL, M. HÖLSCHER and K. LÜCKE \\ Inst. f. Allgemeine Metallkunde and Metallphysik, RWTH Aachen, \\ Kopernikus, Str. 14, D-5100 Aachen, Germany
}

Rolling and recrystallisation textures of $\mathrm{Fe}-3 \% \mathrm{Si}(\mathrm{HiB})$ were investigated in center and subsurface layer by the ODF-method. Degree of cold rolling and recrystallisation time were varied. The results were compared with results obtained for low carbon steel and the mechanisms of texture formation were discussed. At the beginning of the paper the different methods of representation of OVF data were demonstrated.

KEY WORDS Fe-3\% Si, rolling texture, recrystallization texture, texture representation, texture inhomogeneity, low carbon steel.

Textures influence strongly the properties of metallic materials. For example, for the here considered electrical steels on the basis $\mathrm{Fe}-3 \% \mathrm{Si}$ it is very favourable to have the $\langle 001\rangle$ axis in rolling direction (RD), because this crystallographic direction as direction of easiest magnetisation leads to the lowest energy losses in transformers. This condition is fulfilled in practice in the very most cases by applying the Goss texture (Cube on edge) $\langle 001\rangle \|$ RD and $\langle 011\rangle \|$ ND.

There are already several industrial processes able to produce sheets with a very exact Goss texture. They can be divided into two groups. First we have the two-stage process where a hotband containing MnS precipitates (RGO, i.e. Regular Grain Oriented material) is cold rolled in two stages of about $70 \%$ and $50 \%$ reduction with intermediate annealing and then primary recrystallized. The second process applies a hotband containing additionally AIN precipitates (HiB, i.e. High Inductivity material) and cold rolling in one stage to about $85 \%$ followed by the primary recrystallization. In both processes a subsequent secondary recrystallization leads to the Goss orientation as only final texture component, in the second process to an even higher accuracy with deviations of $<5^{\circ}$. It is still astonishing that by simple industrial rolling and annealing procedures such a sharp orientation concentration can be formed.

Although the industrial processing is quite successful, there is little known about the texture development and the underlying mechanisms leading to the final Goss texture by these processes. The present work was carried out to get more information about the formation of rolling and recrystallization textures of the $\mathrm{Fe}-3 \% \mathrm{Si}$ alloys. Here, as an example, the results on $\mathrm{HiB}$ material are reported. In contrast to nearly all former investigations in which pole figures were used except in Ref. 1), here orientation distribution functions (ODFs) with their much higher resolution were applied. As introduction to textures in bcc materials and for comparison, here first the textures of a low carbon steel are briefly reviewed. 


\section{EXPERIMENTAL PROCEDURES AND PRESENTATION OF TEXTURES}

The investigations of a low carbon steel $(0.007 \% \mathrm{C}$, vacuum degassed) started from hot band (HB) of $6.9 \mathrm{~mm}$ thickness. Textures were measured after cold rolling (CR) to $90 \%$ reduction and after a subsequent recrystallization (RX) $\left(700^{\circ} \mathrm{C}, 100 \mathrm{~s}\right)$.

As starting material for the electrical steel a HB of commercial normalized HiB with AlN and MnS precipitates was used. For systematic examination of the development of the cold rolling texture, the HB was rolled to $25 \%, 50 \%$, $70 \%$, $82 \%$, and $85 \%$ rolling reduction on a laboratory mill. The reduction per pass was chosen to ensure homogeneous deformation over the whole thickness of the specimen. ${ }^{2}$ To observe the development of the primary recrystallization texture, a $82 \%$ rolled specimen was annealed isothermally at $700^{\circ} \mathrm{C}$ for 7,15 , and 30 seconds. All specimens were annealed in a salt bath and subsequently water quenched. By this treatment also decarburization was avoided.

Microstructure was examined in transverse and sheet plane using optical and transmission electron microscopy (TEM). For texture measurements the specimen were mechanically grinded and etched with $\mathrm{HF}$ and $\mathrm{H}_{2} \mathrm{O}_{2}$ either down to the center layer at $s=0$ or down to the subsurface layer at $s=0.8$ (the parameter $s$ is defined as the distance of the investigated layer from the center layer divided by the half thickness).

For measurement of texture a fully automated X-ray goniometer applying the back reflection technique leading to incomplete pole figures (up to $85^{\circ}$ ) was used. Since pole figures are often ambiguous in its interpretation, the ODF method was applied (example see Section 3). The ODF-that is the orientation density $f(g)$ as function of the orientation $g$ (here given by the three Eulerian angles $\varphi_{1}, \Phi$, $\varphi_{2}$ )-is calculated by the series expansion method ${ }^{3}$ from the four measured pole figures $\{011\},\{002\},\{112\}$, and $\{013\}$. The so-called "ghost error" of the ODF which is due to the missing of the odd expansion coefficients in ODFs derived from pole figures and which is very grave and definitely needs to be corrected in fcc metals, plays in bcc metals a minor role (cf. Figure 3 ) and can be neglected. ${ }^{4}$ This is because the ODFs of bcc metals are largely composed of low indexed fibers instead of single orientation components as for fcc metals (cf. Figure 3c) and since for these fibers the ghost error disappears.

The ODFs are represented in the orientation space build up by the three Eulerian angles $\varphi_{1}, \Phi, \varphi_{2}$ (e.g. Figure 2c) in sections at constant $\varphi_{1}$ in form of contour lines in multiples of the random density (cf. Figures 3 ). Because of the cubic crystal symmetry and the orthorhombic sample symmetry each orientation occurs three times ${ }^{5}$ in the range $0^{\circ}<\varphi_{1}, \Phi, \varphi_{2}<90^{\circ}$ of the orientation space shown in Figure 2c. Besides some important orientations also some fiber-type texture components which occur in bcc metals are inserted into Figure 2c.

1. $\gamma$ fiber. It runs from the section $\varphi_{1}=0^{\circ}$ at $\Phi=55^{\circ}$ and $\varphi_{2}=45^{\circ}$ along $\varphi_{1}$ and describes all rotations with $\langle 111\rangle \| N D$. It contains the orientations $\{111\}\langle 110\rangle$ at $\varphi_{1}=0^{\circ}$ and $\{111\}\langle 112\rangle$ at $\varphi_{1}=30^{\circ}$. Because of the three-fold symmetry of the $\langle 111\rangle$ axis the parts $\varphi_{1}=0^{\circ}$ to $30^{\circ}, 60^{\circ}$ to $30^{\circ}$ and $60^{\circ}$ to 90 are symmetrically equivalent.

2. $\alpha$ fiber. This fiber is incomplete. It runs from $\varphi_{1}=0^{\circ}, \Phi=0^{\circ}$, and $\varphi_{2}=45^{\circ}$ along $\Phi$ to $\Phi=55^{\circ}$ and describes rotations with $\langle 110\rangle \| R D$. It contains the 
orientation $\{001\}\langle 110\rangle,\{112\}\langle 110\rangle$ and $\{111\}\langle 110\rangle$. The symmetrically equivalent position of this fiber starts at $\varphi_{1}=45^{\circ}, \Phi=90^{\circ}, \varphi_{2}=90^{\circ}$ and runs through the Euler space till $\varphi_{1}=60^{\circ}, \Phi=55^{\circ}, \varphi_{2}=45^{\circ}$.

3. $\eta$ fiber. It runs from $\varphi_{1}, \Phi, \varphi_{2}=0^{\circ}$ along $\Phi$ and describes all rotations around $\langle 100\rangle \mathrm{RD}$. It contains-and that is its importance-the Cube orientations $\{001\}\langle 100\rangle$ and the Goss orientation $\{011\}\langle 100\rangle$.

4. In certain cases also the $\zeta$ and $\varepsilon$ fiber with an $\langle 011\rangle$ axis parallel to ND and TD, respectively, are of interest.

\section{TEXTURES IN LOW CARBON STEEL}

Figure 1 show typical bcc $\{011\}$ pole figures of CR (Figure $1 \mathrm{~b}$ ) and a subsequently recrystallized RX (Figure 1d) low carbon vacuum degassed (VD) steel. The measured intensities are normalized to the random intensity. The interpretation of such pole figures, however, is rather difficult since each orientation gives here 6 reflection spots (cf. Figures 1b,d). For example the two pole figures of Figure 1

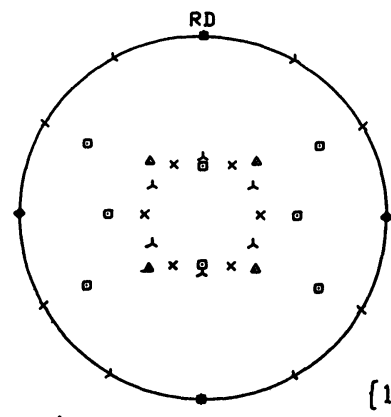

a)
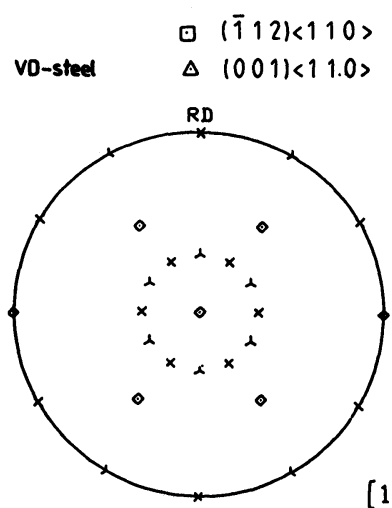

c)

$$
\begin{array}{ll} 
& \times(1 \overline{1} 1)\langle 110\rangle \\
\text { vo-steel } & Y(11 \overline{1})\langle 112\rangle
\end{array}
$$

$\left[\begin{array}{lll}1 & 1 & 0\end{array}\right]$

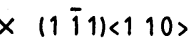

$\times(111)<110\rangle$
$Y \quad(11 \overline{1})<112\rangle$

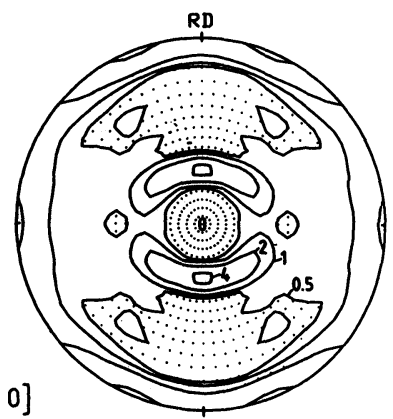

d)

Figure 1 a) Ideal orientation after cold rolling (CR). b) Measured $\langle 110\rangle$ pole figure after cold rolling for a VD steel. c) Ideal orientation after primary recrystallization (RX). d) Measured $\langle 110\rangle$ pole figure after RX of a VD steel. 

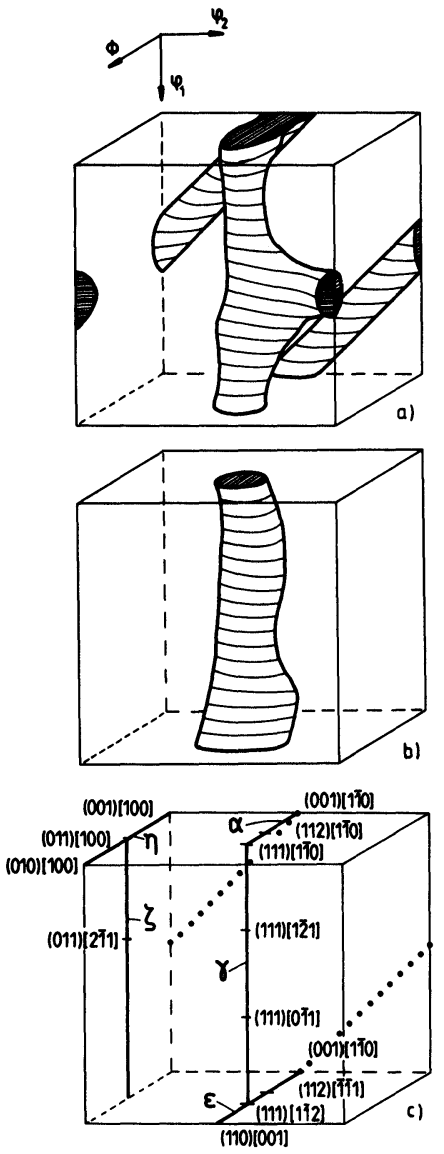

Figure 2 Three-dimensional representation of a) rolling texture and b) primary recrystallization texture with the surface of the inserted orientation bodies corresponding to a density of 5 times random; Figures 1 to 6 all represent the same texture of a VD steel. c) Schematic representation of the main positions and orientation fibers after cold rolling and primary recrystallization.

look rather similar. But, as can be seen from the ideal orientations plotted in Figures 1a, c, the rolling texture can be better described by the orientations $\{001\}\langle 110\rangle,\{112\}\langle 110\rangle,\{111\}\langle 110\rangle$, and $\{111\}\langle 112\rangle$ whereas for the recrystallized texture $\{111\}\langle 110\rangle,\{111\}\langle 112\rangle$, and additionally a very small Goss component $\{011\}\langle 100\rangle$ are necessary.

A much clearer picture arises, if the ODFs are plotted as in Figures $2 a, b$. The surfaces of the bodies shown there indicate the orientations for which the orientation density is just 5 times random. By comparison with Figure $2 \mathrm{c}$ it is to be seen that these two bodies which represent typical bcc textures surround the $\alpha$ and $\gamma$ fiber.

Figures $3 \mathrm{a}, \mathrm{d}$ show these two ODFs in more detail by contour lines in the $\varphi_{1}=$ constant sections. This figure also demonstrates that the ghost error can here be neglected. In Figures $3 \mathrm{~b}$, e the model ODFs are represented which are obtained for the CR and RX case obtained by fitting of peak-type, and fiber-type components with Gaussian scattering (all listed in Table 1) to the measured ODFs, and in Figures 3c, $\mathrm{f}$ the measured ODFs corrected by the odd parts taken from model ODFs (i.e. the approximated true ODFs) are shown. One sees that the fit between measured ODF (Figures. 3a,d) and model (Figures 3b,c) is 

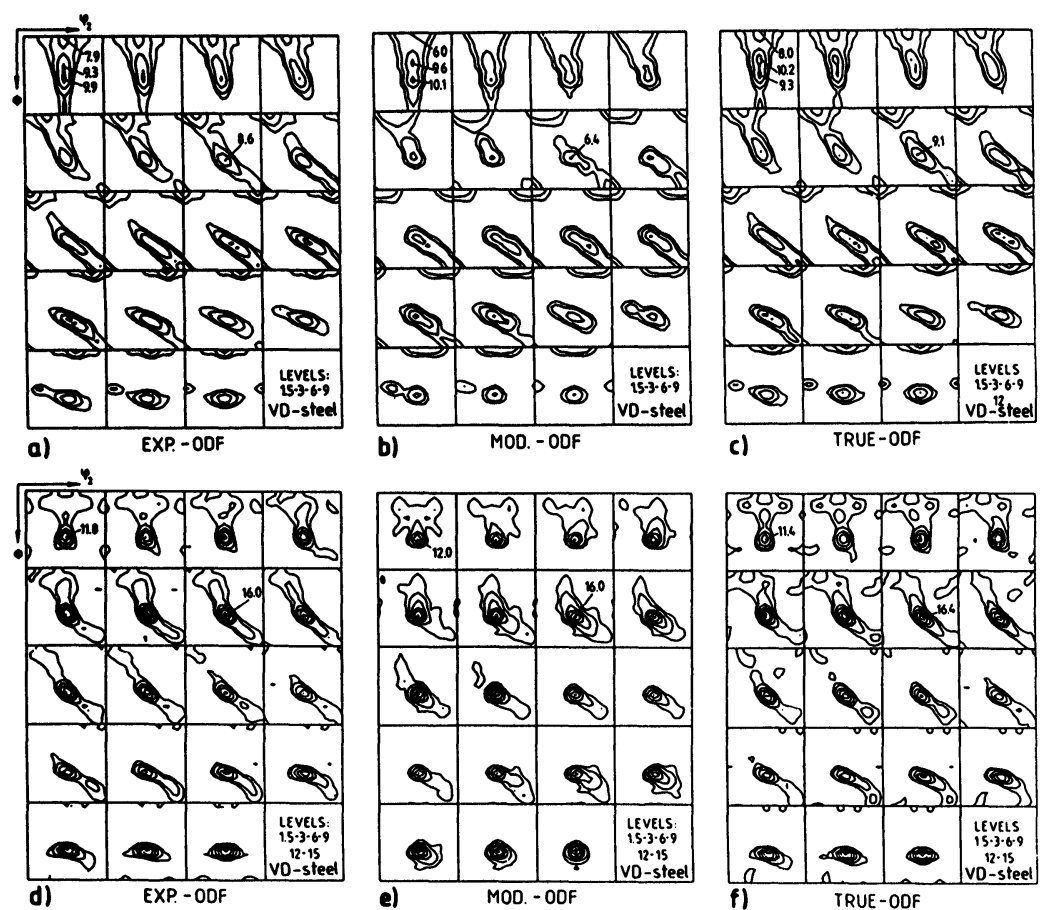

Figure 3 Experimental ODF (a, d), model ODF (b, e) and ghost-corrected ODF (c, f) after cold rolling $(a, b, c)$ and primary recrystallization $(d, e, f)$ of a VD steel.

excellent, but, by comparison between measured and corrected ODF (Figures $3 c, f)$, that the corrections are very minor.

A very compact presentation of such textures yields the $\varphi_{2}=45^{\circ}$ section (Figure 4) since it contains both the $\alpha$ and $\gamma$ fiber and additionally the Goss orientation (Figure 4c). To have still more quantitative information it is useful to plot the orientation densities $f(g)$ along the fiber, i.e. as function of the angle of rotation around the fiber axis, as shown in Figure 5 for the $\alpha, \gamma$, and $\eta$ fiber. This will be used as a standard way for presenting bcc CR and RX textures.

Table 1 Texture component parameters in vacuum degassed steel.

\begin{tabular}{|c|c|c|c|c|c|c|c|c|c|c|c|}
\hline \multicolumn{6}{|c|}{ Cold rolled ( $90 \%$ reduction) } & \multicolumn{6}{|c|}{ Subsequently recrystallized $\left(700^{\circ} \mathrm{C}\right)$} \\
\hline Component & $\varphi_{1}$ & $\Phi$ & $\varphi_{2}$ & $W[\%]$ & $\underset{\left[{ }^{\circ}\right]}{\boldsymbol{\vartheta}}$ & Component & $\varphi_{1}$ & $\Phi$ & $\varphi_{2}$ & $W[\%]$ & $\begin{array}{l}\psi, \vartheta \\
{\left[{ }^{\circ}\right]}\end{array}$ \\
\hline $\begin{array}{r}\text { Background } \\
\{239\}\langle 321\rangle \\
\approx\{001\}\langle 320\rangle \\
\{112\}\langle 110\rangle \\
\{223\}\langle 692\rangle \\
\{111\} \| \text { ND } \\
\langle 110\rangle \| \text { RD }\end{array}$ & $\begin{array}{r}0.0 \\
0.0 \\
0.0 \\
16.5\end{array}$ & $\begin{array}{r}\overline{20.0} \\
6.0 \\
35.0 \\
40.0 \\
- \\
-\end{array}$ & $\begin{array}{l}34.0 \\
33.0 \\
45.0 \\
43.5\end{array}$ & \begin{tabular}{r|}
15.6 \\
3.0 \\
13.3 \\
9.9 \\
14.8 \\
26.9 \\
16.0
\end{tabular} & $\begin{array}{r}- \\
4.8 \\
10.3 \\
9.5 \\
7.7 \\
8.1 \\
7.0\end{array}$ & $\begin{array}{c}\text { Background } \\
\approx\{322\}\langle 274\rangle \\
\{438\}\langle 742\rangle \\
\{129\}\langle 210\rangle \\
\{113\}\langle 110\rangle \\
\{223\}\langle 110\rangle \\
\{111\} \| N D\end{array}$ & $\begin{array}{r}33.0 \\
24.0 \\
0.0 \\
0.0 \\
0.0\end{array}$ & $\begin{array}{l}-\overline{60.0} \\
32.0 \\
15.0 \\
25.0 \\
42.5 \\
-\end{array}$ & $\begin{array}{l}55.0 \\
41.0 \\
25.0 \\
45.0 \\
45.0\end{array}$ & $\begin{array}{r}2.3 \\
47.7 \\
5.2 \\
10.7 \\
4.3 \\
3.0 \\
36.6\end{array}$ & \begin{tabular}{r|}
11.0 \\
8.5 \\
6.1 \\
7.4 \\
6.3 \\
5.9
\end{tabular} \\
\hline
\end{tabular}



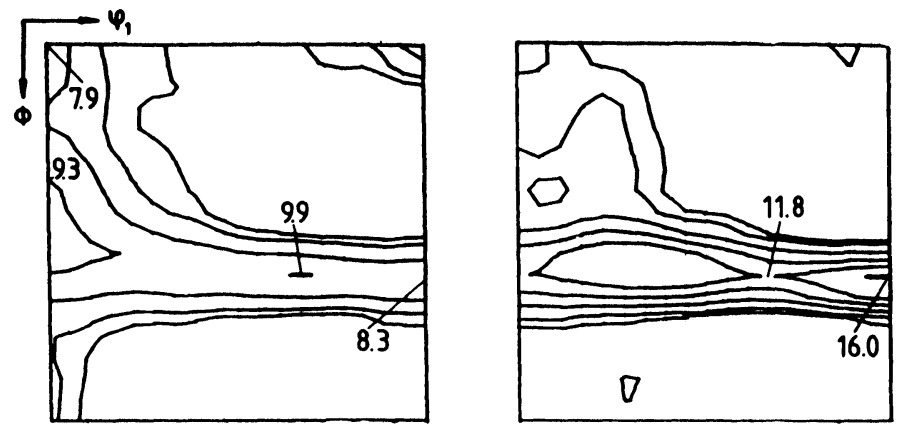

VD-steel

a)

b)
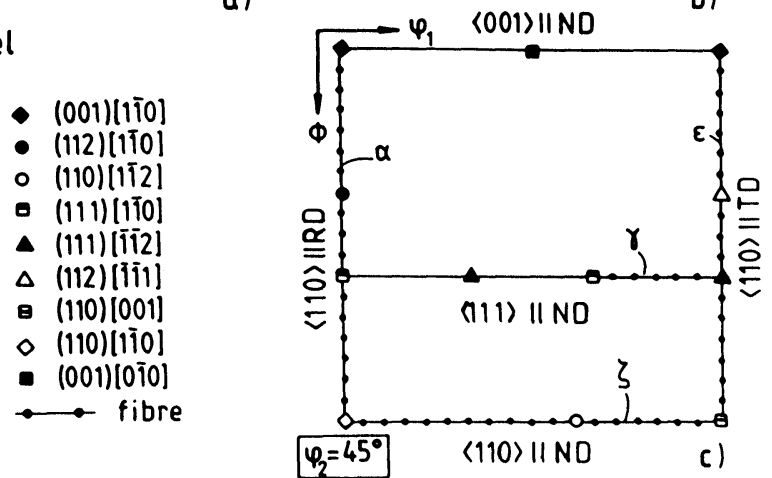

Figure $4 \varphi_{2}=45^{\circ}$ sections through the ODFs: a) after cold rolling and b) after primary recrystallization of a VD steel; c) Ideal positions and fibers.

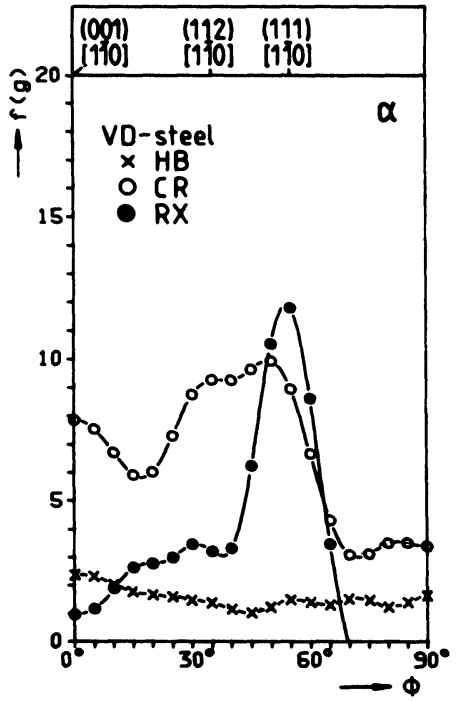

$\langle 110\rangle \| R D$
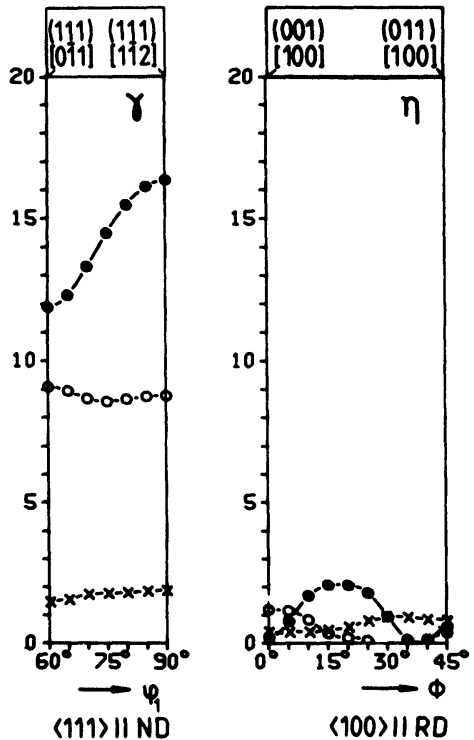

Figure $5 \alpha$ fiber, $\gamma$ fiber and $\eta$ fiber; hot band and after cold rolling and primary recrystallization of a VD steel. 

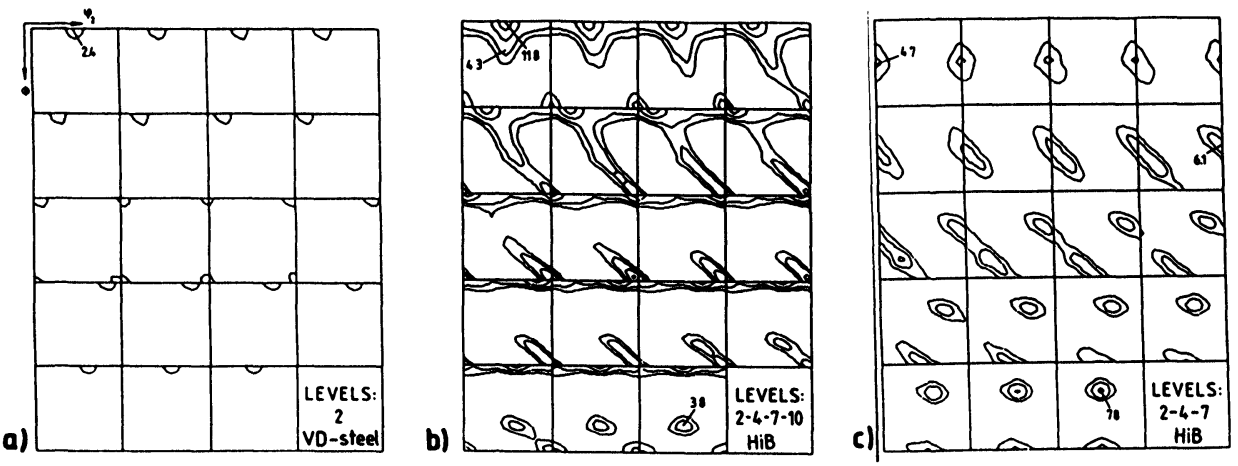

Figure 6 Texture of hot band (HB) before cold rolling; a) VD steel; b) HiB steel, center layer $s=0$; c) $\mathrm{HiB}$ steel, near-surface layer $(s=0.8)$.

Coming to the results it is found that the HB of this VD steel is practically textureless throughout the thickness; Figure 5 (crossed symbols and Figure 6a) show only a very weak $\{001\}\langle 110\rangle$ orientation. The CR rolling texture to be seen in Figures 3a, 4a, 5 (open symbols) can be described best by the components of the $\alpha$ and $\gamma$ fiber, particularly by $\{001\}\langle 110\rangle,\{112\}\langle 110\rangle,\{111\}\langle 110\rangle$, and $\{111\}\langle 211\rangle$. By RX after such high rolling degrees (Figures $3 d, 4 b, 5$ ) the orientation density of the $\alpha$ fiber nearly completely decreases (except for $\{111\}\langle 110\rangle$ where the $\alpha$ and $\gamma$ fibers join) and the density of the $\gamma$ fiber increases, especially at $\{111\}\langle 211\rangle$. Additionally an increase on the $\eta$ fiber near the Goss orientation $\{011\}\langle 100\rangle$ can be recognized, which will become important for discussing the results of the electrical steels.

\section{HOTBAND, RESULTS AND DISCUSSION}

The ODFs for annealed HiB hotband in center $(s=0)$ and sub-surface layer $(s=0.8)$ are presented in Figures $6 \mathrm{~b}, \mathrm{c}$. In contrast to the HB texture of the VD steel (Figure 6a) which is nearly random, the present $\mathrm{HB}$ of $\mathrm{HiB}$ exhibits very strong textures with, moreover, a strong through-thickness variation. One finds a pronounced sub-surface texture with a maximum at $s=0.8$ (Figure 6c) which between $s=0.6$ and $s=0.3$ rotates into the texture of the center layer at $s=0$ (Figure 6b). A more detailed description is given in Figure 7 where besides the $\alpha$, $\gamma$, and $\eta$ fiber also the $\zeta$ and $\varepsilon$ fiber are shown. The sub-surface texture at $s=0.8$ (open symbols) is mainly characterized by single peaks around $\{011\}\langle 211\rangle$ ( $\zeta$ fiber), $\{112\}\langle 111\rangle$ ( $\varepsilon$ fiber), and $\{011\}\langle 100\rangle(\eta, \xi, \varepsilon$ fiber). The texture in the center layer (filled symbols) consists of the partial $\alpha$ fiber with the main component $\{001\}\langle 110\rangle, \gamma$ with nearly $\{111\}\langle 211\rangle$ (better described by $\{81111\}\langle 1144\rangle$ as to be seen at $\varepsilon$ fiber $\left.\varphi_{1}=55^{\circ}, \Phi=63^{\circ}, \varphi_{2}=45^{\circ}\right)$ and $\eta$ with $\{001\}\langle 100\rangle$. The other pronounced peaks in Figure 7 are only symmetrical equivalent to those listed above.

Concerning the interpretation of the HB textures of the HiB material, one recognizes that for $s=0$ those components obtained by cold rolling appear (see 


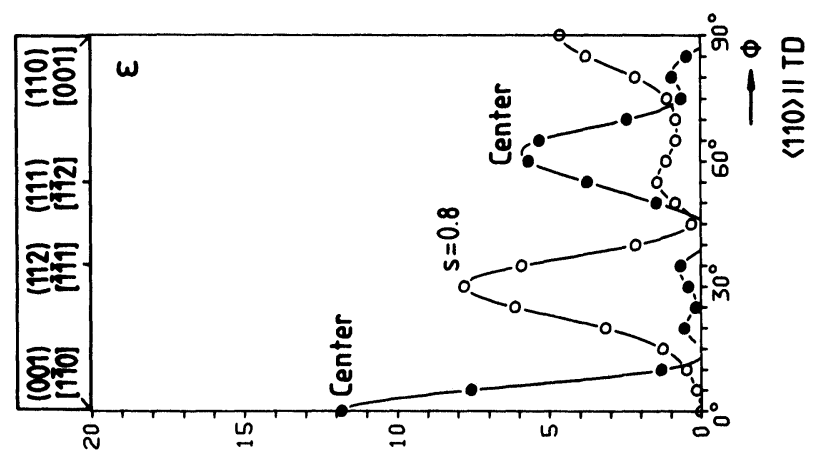

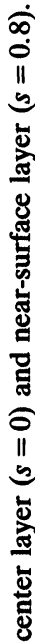
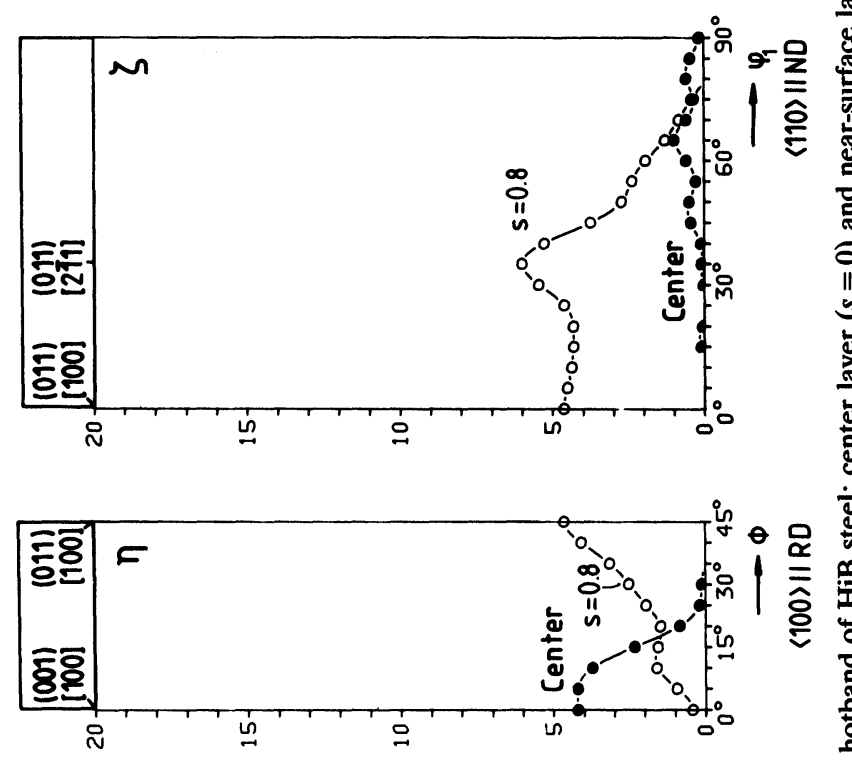

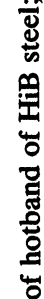
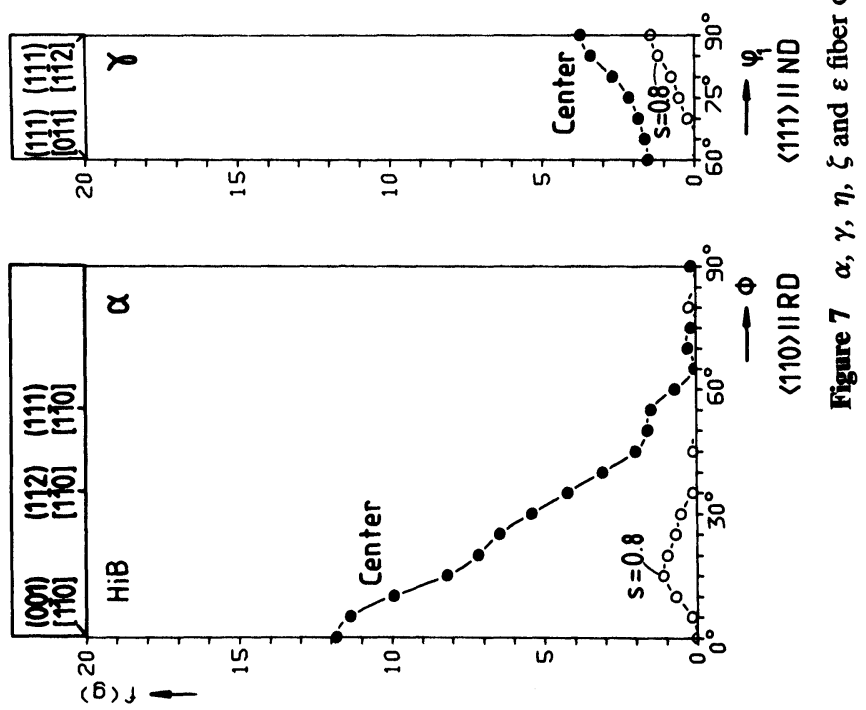
Section 5), namely those of the $\alpha$ and $\gamma$ fiber. But there are obvious deviations from the normal $\mathrm{CR}$ textures of $\mathrm{HiB}$ (cf. Section 5), especially a very strong $\{001\}\langle 110\rangle$ as only component on the $\alpha$ fiber, a rather low $\gamma$ fiber and a shift of the ideal $\{111\}\langle 211\rangle$ to higher $\Phi$ values towards $\{81111\}\langle 1144\rangle$. This is not understood in detail, but appears to be connected to the very inhomogeneous hot rolling condition. It is observed that different hot rolling conditions (e.g. different amounts of reduction per pass) lead to quite different hotband textures even in the center layer. ${ }^{6}$ This is also found for the carbon steel (VD) where only a small $\{001\}\langle 110\rangle$ component appears (Figure 6a).

For $s=0.8$, in contrast, the observed orientations are all such which are stable under shear. This demonstrates a strong shear deformation near the surface during hot rolling. It is astonishing that even after the annealing treatment in both layers the corresponding hot rolling texture appears. Apparently dynamic recovery during hot rolling of $\mathrm{HiB}$ prevents that a high dislocation density responsible for recrystallization is formed. Furthermore, the $\alpha \rightarrow \gamma \rightarrow \alpha$ phase transformation which is the reason for destroying the deformation texture of the HB in low carbon steel (see Section 3 ) is suffered here only by a small part of the material.

\section{COLD ROLLING: RESULTS AND DISCUSSION}

Also the CR texture will be described for the layers $s=0$ and $s=0.8$. For the center layer (Fig. 8) CR causes an increase of $f(g)$ along the $\alpha$ and $\gamma$ fiber, but differently for rolling reductions up to $70 \%$ and beyond $70 \%$. Up to $70 \%$ the $\gamma$ fiber increases homogeneously and beyond a distinct peak at $\{111\}\langle 110\rangle$ is
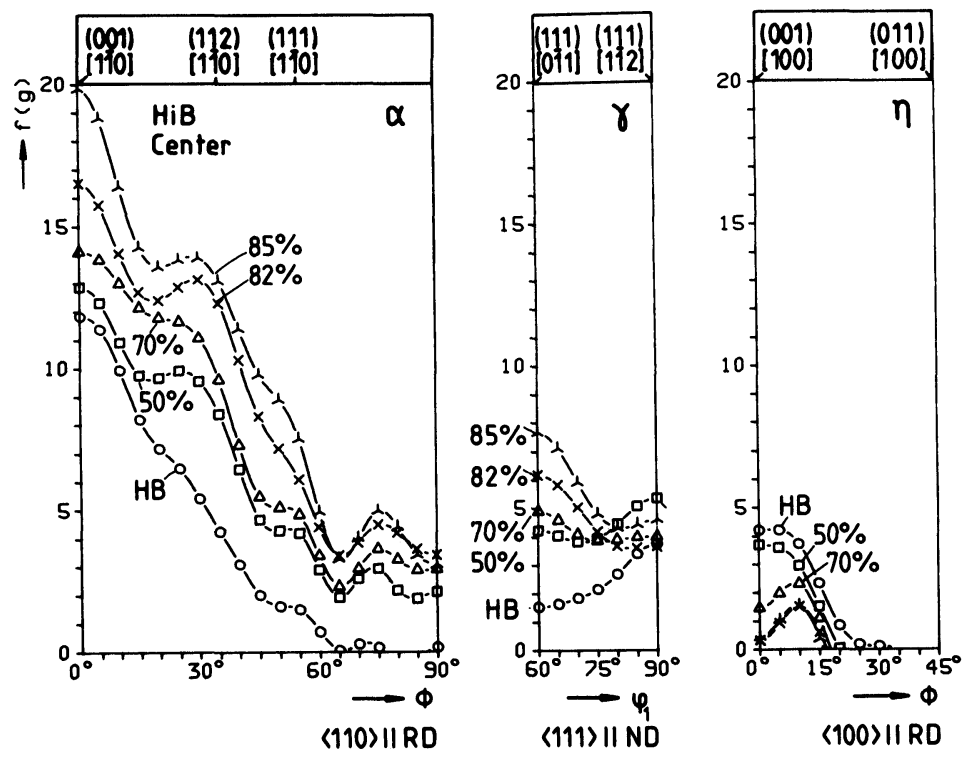

Figure $8 \alpha, \gamma$, and $\eta$ after different degrees of cold rolling of HiB steel; center layer $(s=0)$. 


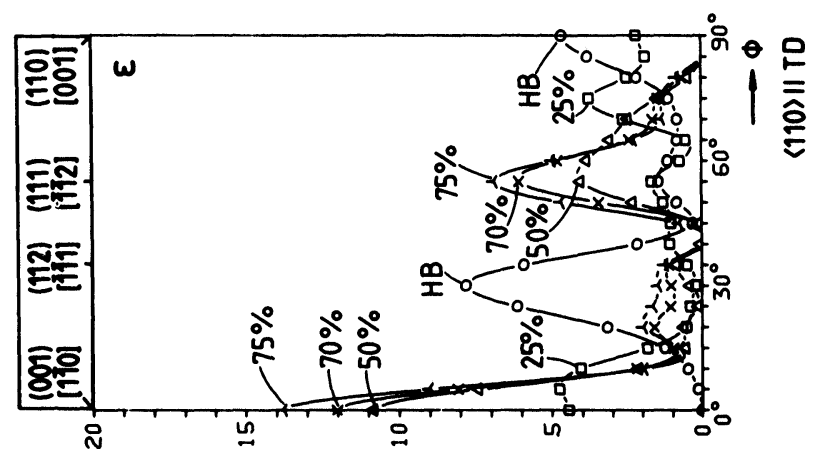

我
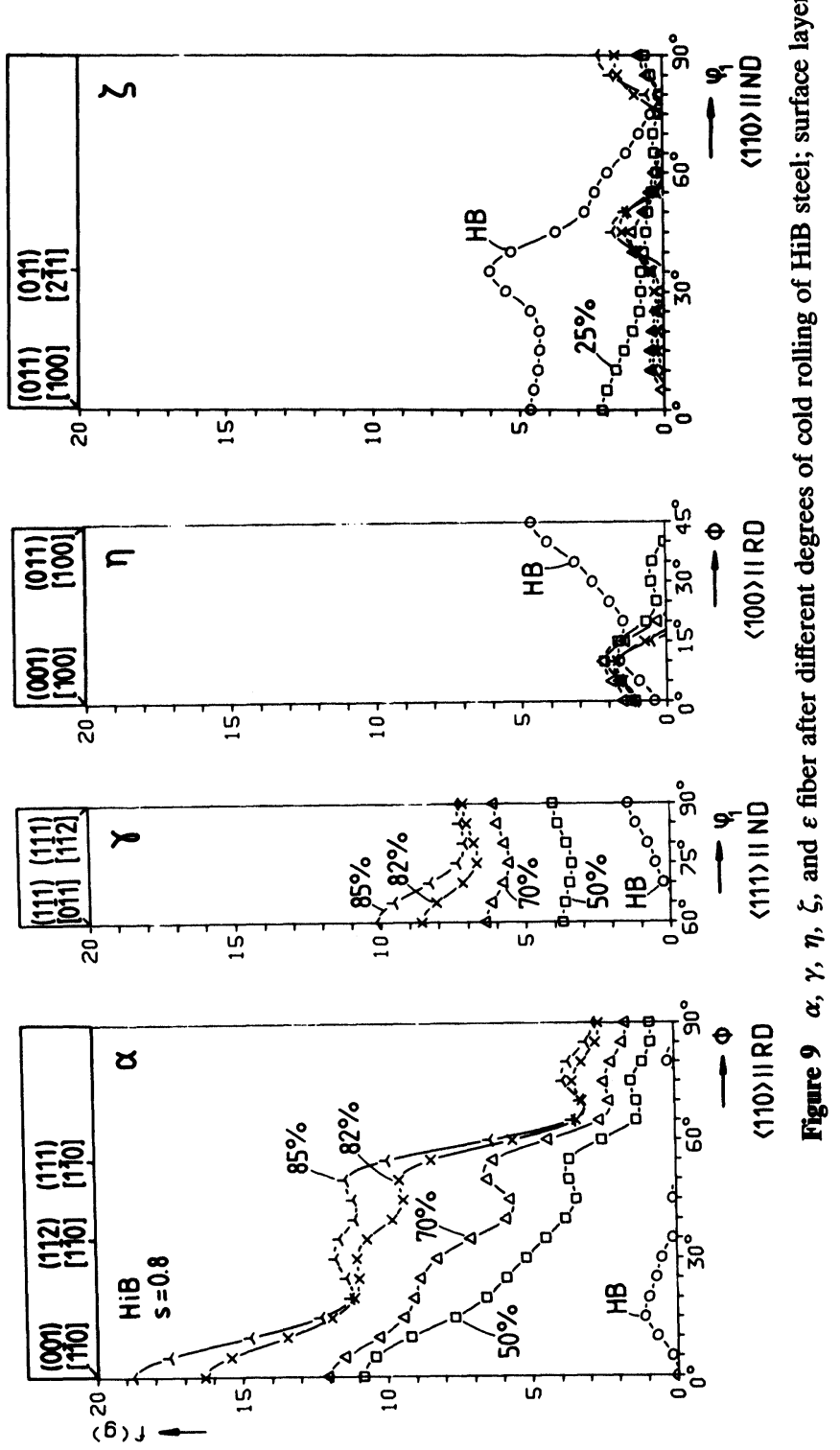
formed. On the $\alpha$ fiber up to $70 \%$ reduction the pronounced maximum of $\{001\}\langle 110\rangle$ due to $\mathrm{HB}$ will be strengthed whereas further rolling leads to a predominant increase of $\{112\}\langle 110\rangle$ and $\{111\}\langle 110\rangle$. For the layer $s=0.8$ (Figure 9) for which all 5 main fibers are presented the $\alpha$ and $\gamma$ fiber densities increase similar as in the $s=0$ plane, but more strongly. Already a deformation of $25 \%$ leads there to a nearly complete disappearance of the shear components. They rotate from orientations stable for shear into those stable for rolling. For example, as can be seen on the $\varepsilon$ fiber, $\{011\}\langle 100\rangle\left(\Phi=90^{\circ}\right)$ rotates around the axis $\langle 011\rangle \|$ TD into both variants of $\{111\}\langle 211\rangle\left(\Phi=55^{\circ}\right)$ and $\{112\}\langle 111\rangle$ splits into $\{001\}\langle 110\rangle\left(\Phi=0^{\circ}\right)$ and $\{111\}\langle 211\rangle$. These results are in good agreement with single crystal experiments which also exhibit these rotations. ${ }^{7}$ As general result-which can be seen by comparing Figures 8 and 9-the ODF at $s=0.8$ becomes qualitatively similar to that at $s=0$ but is less pronounced, i.e. the $\alpha$ and $\gamma$ fiber densities are smaller than at $s=0$.

The general tendencies of texture development of bcc metals during CR can largely be interpreted by Taylor-type theories with full and relaxed constraints. ${ }^{8,9}$ For low deformation when the grains are still of globular shape, Taylor's original assumption that each grain experiences the same strains, i.e. as the specimen at the whole (full constraints) tensile strains || RD and compressional strains || ND, works quite well. Calculations on this basis predict the partial $\alpha$ fiber running from $\{001\}\langle 110\rangle$ to $\{112\}\langle 110\rangle$ and a fiber called $\beta$ fiber, which is running from $\{112\}\langle 110\rangle$ to $\{81111\}\langle 1144\rangle$. However, this $\{81111\}\langle 1144\rangle$ orientation being related to $\{111\}\langle 211\rangle$ by a $8^{\circ}$ rotation around $\langle 110\rangle \|$ TD seldom appears, but instead $\{111\}\langle 211\rangle$ is found. This is predicted by the relaxed constraints models. If, by cold rolling, the grains have assumed the shape of thin bands, the incompatibilities for shear parallel rolling direction ("lath model") and, after further rolling, for shear parallel transverse direction ("pancake model") are relaxed. ${ }^{9}$ Indeed, in good agreement with the experimental results, calculations based on the first model lead to the observed peaks at $\{111\}\langle 211\rangle$ and $\{112\}\langle 110\rangle$ and on the second model to the peak at $\{111\}\langle 110\rangle$ occurring at high deformations. This means the above mentioned limit deformation of about $70 \%$ reduction seems to indicate the limit between lath and pancake model.

\section{RECRYSTALLIZATION: RESULTS AND DISCUSSION}

$\mathrm{RX}$ is here characterized by a disappearance of the sharp rolling texture (crossed symbols) and remaining of a rather weak texture after full recrystallization (filled triangles). As for the CR textures also for the RX textures the two layers at $s=0$ and $s=0.8$ will be considered.

At $s=0$ (Figure 10) short annealing times $(7 \mathrm{~s})$ lead to a slight increase in the density in the $\alpha$ fiber which can be explained by recovery effects. The occurrence of recrystallization (full symbols) is marked by a strong density drop along the $\alpha$ fiber, by a drop of $\{111\}\langle 110\rangle$ and an increase of $\{111\}\langle 211\rangle$ on the $\gamma$ fiber and by a formation of a small orientation density along $\eta$ fiber, but with negligible intensity at the Goss orientation $\{011\}\langle 100\rangle$. For $s=0.8$ (Figure 11) a drop in both $\alpha$ and $\gamma$ fiber takes place already after $7 \mathrm{~s}$, i.e. here recrystallization starts earlier. After further annealing also here the change from $\{111\}\langle 110\rangle$ to 

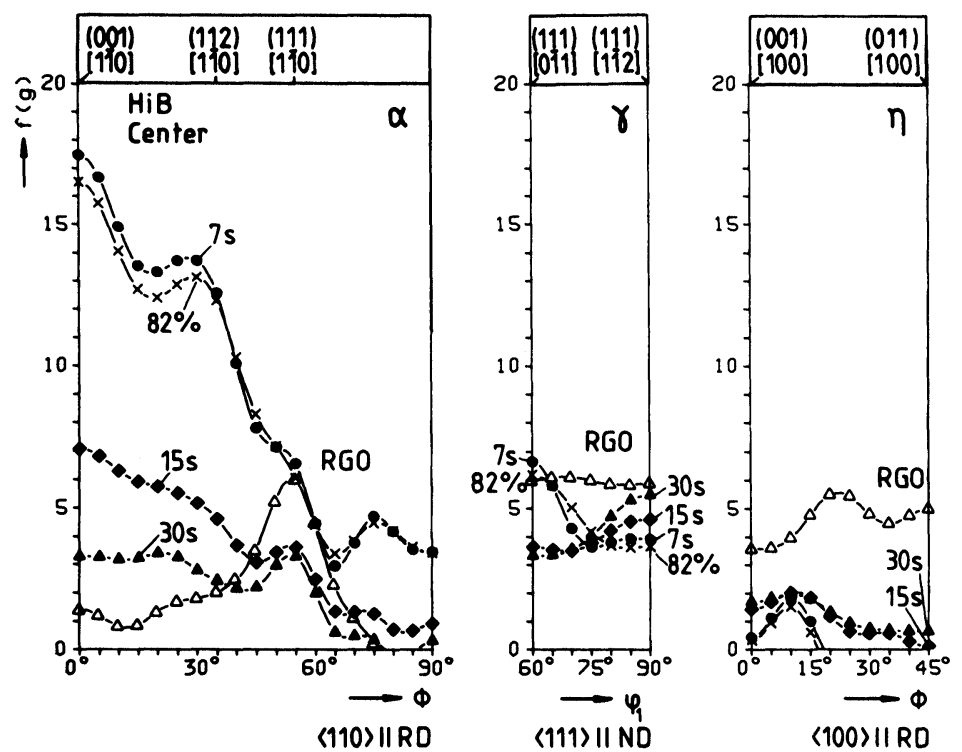

Figure $10 \alpha, \gamma$, and $\eta$ fiber after different annealing times at $700^{\circ} \mathrm{C}$ of $\mathrm{HiB}$ steel cold rolled to $82 \%$ reduction; center layer $(s=0)$.

$\{111\}\langle 211\rangle$ in the $\gamma$ fiber occurs. Additionally a density increase along the whole $\eta$ fiber including $\{011\}\langle 100\rangle$ can be observed.

Concerning the interpretation it is mostly assumed that the $\gamma$ fiber grains have a higher dislocation density and thus a higher rate of subgrain growth finally leading to $\{111\}$ nuclei. Moreover, the $\{111\}\langle 211\rangle$ orientations of this fiber have an orientation relationship of $a \approx 32^{\circ}$ rotation around $\langle 110\rangle$ which is close to the ideal relationship for maximum growth rate for bcc metals of $27^{\circ}\langle 110\rangle .^{11}$ The observed decrease of the fiber and increase of $\{111\}\langle 211\rangle$ during recrystallization (Figures 10,11) can thus be explained as oriented growth of $\{111\}\langle 211\rangle$ nuclei into $\{112\}\langle 110\rangle$. For the Goss orientation the mechanisms of nucleation and growth during primary RX are still in discussion although it is largely accepted that formation of shear bands in $\{111\}\langle 211\rangle$ crystals during $\mathrm{CR}$ plays an important role (e.g. Ref 10). In these shear bands frequently Goss orientations which might be able to act as nuclei are found and the orientation relationship of $35^{\circ}$ rotations around $\langle 110\rangle$ to the $\{111\}\langle 112\rangle$ matrix is again close to the ideal relationship of $27^{\circ}\langle 110\rangle$. The observed decrease of $\{111\}\langle 211\rangle$ and increase of $\{011\}\langle 100\rangle$ together with other orientations along the $\eta$ fiber as to be seen in Figure 11 is in good agreement with this assumption of nucleation of $\{011\}\langle 100\rangle$ in the shear bands of $\{111\}\langle 211\rangle$ crystals and preferred growth into this orientation.

\section{GENERAL DISCUSSION AND CONCLUSION}

1. In investigating the detailed texture development in $\mathrm{HiB} \mathrm{Fe}-3 \% \mathrm{Si}$ steels the ODF method with its high resolution proves itself to be far superior to the pole 


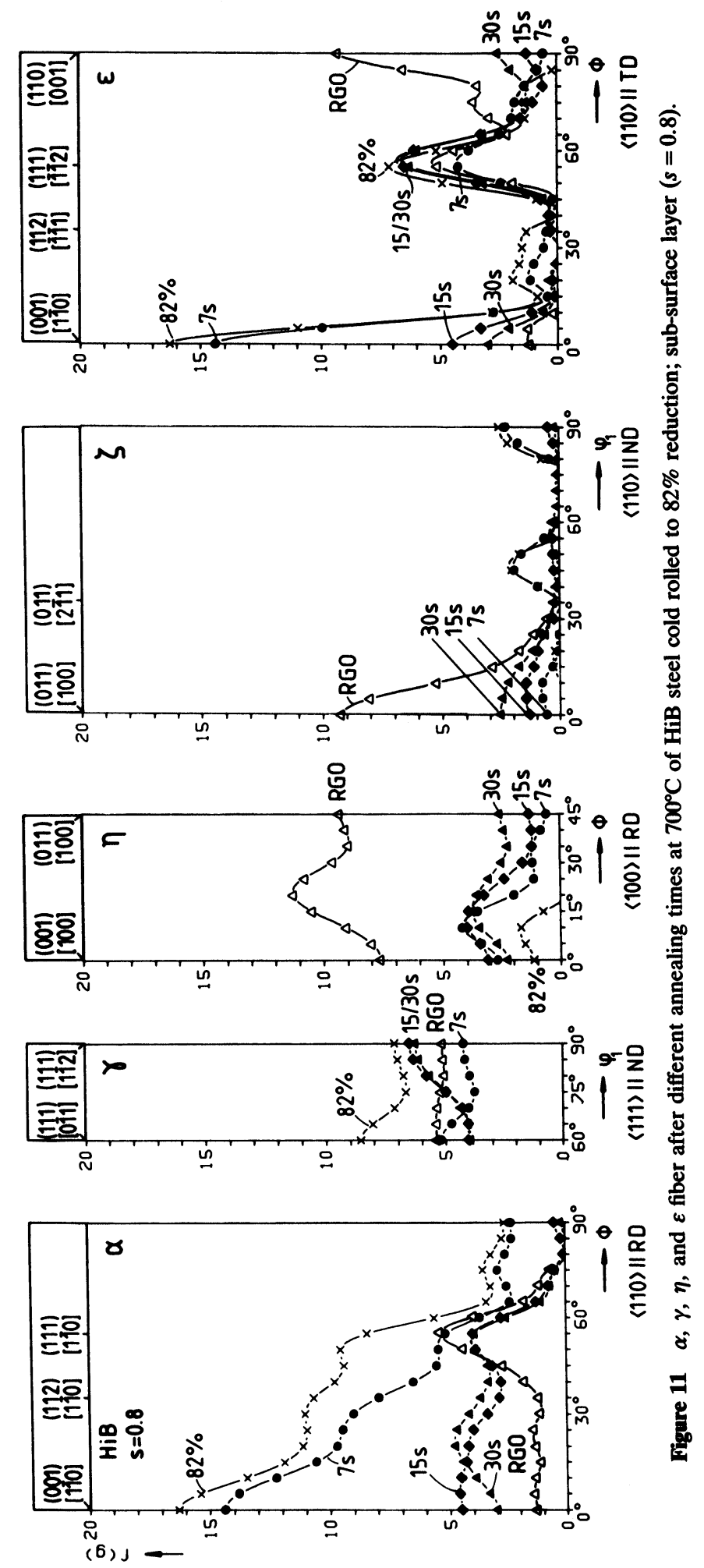


figure method. Because of the fiber character of the resulting textures the ghost error is here rather small.

2. Even though one has to take into account large differences in the starting texture, the general features of texture development by cold rolling and annealing in the center layer of the present HiB and VD steel are similar. They consist in (i) a build up of a partial $\alpha$ fiber and complete $\gamma$ fiber during cold rolling which is also predicted as texture after plane strain compression by application of the full constraints and relaxed constraints Taylor-type theories, and (ii) a sharp drop of $\alpha$ fiber and remaining of $\gamma$-fiber during primary recrystallization which is here interpreted by oriented nucleation and oriented growth type points of view. The main differences occurring for different bcc steels are in the recrystallization texture and concern the absolute height of the orientation density in the $\gamma$ fiber, the density distribution along the $\gamma$ fiber (between $\{111\}\langle 110\rangle$ and $\{111\}\langle 211\rangle$ ) and, most important, the formation of a strong $\eta$ fiber with Goss orientation $\{011\}\langle 100\rangle$ (which is more pronounced in the HiB steel).

3. In order to compare the present $\mathrm{HiB}$ material with the regular grain oriented (RGO) material containing only MnS-inhibitors, the $f(g)$ values for complete primary recrystallization texture are inserted into Figures 10 and 11 (open triangles). One sees that the HiB-material with its higher reduction and sharper cold rolling texture shows a significant lower orientation density at $\{011\}\langle 100\rangle$. This primary recrystallization texture with sharper $\{111\}\langle 112\rangle$ and lower Goss seems to be the reason for a more pronounced selective growth in $\mathrm{HiB}$ and a resulting sharper final Goss orientation after secondary recrystallization.

4. The main difference in the texture of the HiB and low carbon steels lies in a strong texture inhomogeneity across the sheet thickness for the first and the rather homogeneous texture for the second material. The inhomogeneity in the $\mathrm{HiB}$ steel is due to the formation of a shear texture in the sub-surface layers during hot rolling, whereas the low carbon steels-in contrast to HiB-undergo

a)

CR

HB

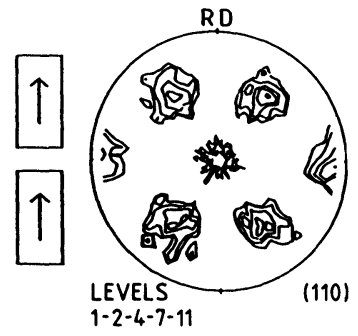

RGO

CR

HB

b)

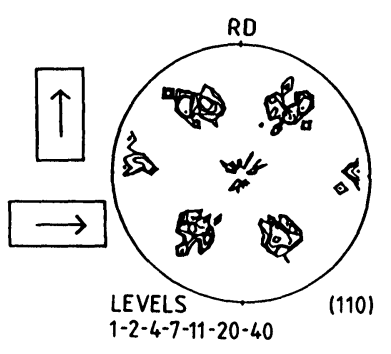

Figure 12 RGO material after secondary recrystallization (according to Lee and Lücke). ${ }^{12}$ a) direction of hot and cold rolling parallel; b) direction of hot and cold rolling perpendicular (for ideal Goss orientation see Figure 1c). 
an $\alpha \rightarrow \gamma \rightarrow \alpha$ transformation during hot rolling by which the previous textures are erased. At cold rolling the shear texture rotates mainly into $\{111\}\langle 211\rangle$ and thus originates a strong $\gamma$ fiber, sometimes even stronger than that of the center layer.

5. As a further consequence of the different hot band textures the $\eta$ fiber in HiB steels reveals a distinct peak at $\{011\}\langle 100\rangle$ after primary recrystallization, in contrast to low carbon steels. This leads to the so called theory of texture inheritance. ${ }^{1}$ The existence of Goss texture in the sub-surface layers after hot rolling was there assumed to be an unconditional prerequisite for development of secondary Goss grains. But experiments of Lee and Lücke ${ }^{12}$ showed that even with cold rolling in $90^{\circ}$ to the hot rolling direction a strong Goss texture would occur during secondary recrystallization (Figure 12), although-looking now in $90^{\circ}$ to the hot rolling direction-the starting (i.e. hot rolling) texture did not longer contain any Goss orientation. This means that no Goss orientations could be inherited and hence other processes, probably shear band formation in $\{111\}\langle 211\rangle$ grains, must be reponsible for the final Goss orientation in electrical steels.

\section{ACKNOWLEDGEMENT}

The authors acknowledge the financial support by the Arbeitsgemeinschaft Industrieller Forschungsvereinigungen (AIF).

\section{References}

1. Mishra, S., Därmann, C. and Lücke, K. (1984). Acta Met. 32, 2185.

2. Asbeck, H. O. and Mecking, H. (1978). Met. Sci. and Eng. 34, 111.

3. Bunge, H. J. (1974). Mathematische Methoden der Texturanalyse, Akademie Verlag, Berlin.

4. Jura, J. and Lücke, K. (1987). Schlippenbach, U.v., ICOTOM 8, Santa Fe.

5. Hansen, H., Pospiech, J., Lücke, K., (1978). Tables for Texture Analysis of Cubic Crystals, Springer-Verlag Berlin.

6. Matsuo, M., Sakai, T. and Suga, Y. (1986). Met. Trans. A 17A, 1313.

7. Taoka, T., Furubayashi, E. and Takeuchi, S. (1966-7). Transactions ISIJ 6, 290 and 7, 95.

8. Van Houtte, P. (1984). Proc. ICOTOM 7, 7, Netherlands.

9. Fortunier, R. and Hirsch, J. (1988). Theoretical Techniques of Texture Analysis, DGM-Verlag.

10. Haratani, T., Hutchinson, W. B., Dillamore, I. L. and Bate, P., (1984). Met. Sci. $18,57$.

11. Ibe, G. and Lücke, K. (1968). Archiv für das Eisenhüttenwesen 39, 693.

12. Lee, H. G. and Lücke, K. (1987). Proc. ICOTOM 8, 643, Santa Fe. 